\title{
An Isolated Posterior Capsule Rupture after Blunt Eye Injury: Management of Traumatic Cataract Case
}

\author{
Eriks Elksnis $^{1}$, Juris Vanags ${ }^{1}$, Eva Dručka ${ }^{2}$, Oskars Gertners ${ }^{1}$, and Guna Laganovska ${ }^{1}$ \\ ${ }^{1}$ Paul Stradins Clinical University Hospital \\ ${ }^{2}$ Riga Stradins University
}

July 29, 2020

\begin{abstract}
The present case report discusses about a male patient with closed-globe traumatic cataract and isolated posterior capsule rupture. There are limited studies reporting about such type of complication. The management with phacoemulsification and combined pars plana vitrectomy with intraocular lens reverse optic capture was performed.
\end{abstract}

\section{Key clinical message:}

Isolated lens posterior capsule rupture after blunt eye injury is a rare complication and demands a special surgical management strategy in order to achieve good visual outcome.

Introduction :

Traumatic cataract with isolated posterior capsule rupture is rare complication of blunt ocular trauma and therefore, demands a well-considered and diverse approach in the surgical management in order to achieve good visual outcome [1][2]. Furthermore, there are few literary sources reporting about such type of complication [1][2][3][4][5].

Case History:

The present case report discusses about a 39-year-old male patient who visited an ocular emergency doctor working in the ophthalmology department on August 14, 2019. The patient complained of gradual visual loss in the left eye, blurred vision, and difficulty in reading small-sized text on the phone or tablet. The complaints mentioned were noticed after a blunt left eye injury at the end of July 2019--more than three weeks before visiting the ophthalmologist--which happened while repairing a tractor. A rubber belt ruptured and hit the patient's left eyeball with high speed and force.

Examination

Ophthalmic examination indicated reduced visual acuity in the left eye. The uncorrected visual acuity (UCVA) was 20/16 in the right eye and 20/40 in the left one. The intraocular pressure (IOP) did not differ significantly between the eyes, which were as follows: $16 \mathrm{mmHg}$ in the right eye and $18 \mathrm{mmHg}$ in the left eye.

An examination of the right eye by the application of a slit lamp was unremarkable. The left eye presented normal periorbital and ocular surfaces. Biomicroscopy revealed no signs of open globe injury. In contrast, it demonstrated smooth and clear cornea, and normal anterior chamber depth with no signs of hyphema or 
presence of cells into the anterior chamber; the pupil was round and had normal pupillary reflex to the bright light. The anterior capsule of the lens was intact, yet isolated, and longitudinal posterior capsule rupture (PCR) with posterior pole lens opacification was recognised (Figure 1). It is a rare complication of a blunt ocular trauma and thus, demands a well-considered and diverse approach in the surgical management [1][3]. The results from gonioscopy revealed a wide angle with no angle recession. Ultrasound B-scan detected flat retina and clear vitreous. Computed tomography scan for skull fractures was negative.

\section{The surgical management}

The present traumatic cataract case was treated in our department. The procedure implied the management of the loss of posterior capsule support with reverse optic capture. The cataract surgery was performed combined with $23 \mathrm{G}$ pars plana vitrectomy. A conventional $2.4 \mathrm{~mm}$ clear corneal incision was made along with paracentesis. A $360^{\circ}$ intact continuous curvilinear capsulorhexis (CCC), smaller than usual--with a diameter of about $4 \mathrm{~mm}$--was created by the use of capsulotomy forceps. Instead of a conventional cataract surgery, the hydrodelineation of the lens nucleus was performed without hydrodissection. The lens nucleus was extracted with low flow settings of a phacomachine. In order to remove the cortex, slow aspiration with manual coaxial handpiece was performed. When the majority of the cortex was removed, an extensive PCR was observed. It excluded the possibility of intraocular lens (IOL) implantation in the bag. To minimise the vitreous prolapse, an injection of dispersive viscoelastic was administered to create a tamponade near the torn part of the capsule. A single-piece foldable IOL was implanted and stable reverse rhexis fixation was obtained by applying a forward optic capture technique. After stabilising the anterior chamber and hydrating the corneal incisions, 23G pars plana vitrectomy was performed to evacuate herniated vitreous from the posterior capsule tear and dropping cortical fragments from the vitreous body. A standard postoperative medication, according to the praxis of the clinic, consists of topical dexamethasone and chloramphenicol. Consequently, it was prescribed and gradually tapered over the period of 4 weeks.

\section{The Outcome}

Six months after the surgery, UNVA was $20 / 25$, while the best corrected visual acuity (BCVA) with a slight myopic shift of $0.5 \mathrm{D}$ was $20 / 16$. The IOP registered was $17 \mathrm{mmHg}$ in both eyes. No signs of active inflammation during slit lamp examination were observed. The implanted IOL was stable and demonstrated no signs of dislocation (Figure 2). A mild stretch of capsular opening was noticed. Optical coherence tomography scans of the anterior segment were performed to visualise the position of the IOL (Figures 3. and 4).

\section{Discussion:}

Blunt ocular trauma may damage cornea, sclera, retina, and subluxate or luxate lens. However, there are few literary sources on isolated PCR following blunt eye injury [2].

The management of PCR depends on several parameters like the extent and location of the tear, the amount of residual nucleus and cortex, and the presence of vitreous in the anterior chamber. As there are limited articles reporting capsular tear fibrosis and successful IOL implantation in the bag [2], the primary plan regarding this case was the IOL implantation in the capsular bag. Considering the extension of the PCR and prolapsed vitreous in the tear, pars plana vitrectomy with lensectomy was selected as the most appropriate method for the treatment of the issue.

Another important point is the selection of the IOL. In case of PCR with the loss of posterior capsule support, many surgeons would consider a 3-piece IOL designed for the sulcus fixation. Unfortunately, such an option was not available in our clinic. Although iris-fixated IOL is one of the options, it must be remembered that most of the lenses of this design are single-piece polymethyl methacrylate (PMMA) IOL and the total length of the lens is $8.5 \mathrm{~mm}$ with an optic of 5 or $6 \mathrm{~mm}$ in diameter. As a result, these lenses are not foldable and require a wide corneal incision and sutures inducing astigmatism[6]. There is no denying that this was not considered to be the best treatment for a 39-year-old male. The surgeons should remember that if the reverse optic capture does not work, it is possible to remove the lens and replace it with a 3 -piece IOL with sulcus 
fixation or iris-fixated IOL. However, if none of this is available, and the only option is a single-piece IOL, a reverse optic capture or so-called forward optic capture can be considered. Reverse optic capture has been mentioned in a previous study during the treatment of similar cases[4]. Reverse optic capture is a technique of IOL implantation during which the haptics of a 3-piece or single-piece lens are positioned posterior to the anterior capsulotomy, while the optic is anterior to the edge of the anterior capsular opening. This technique requires that the anterior capsular rim of the CCC is intact for the full 360 degrees and smaller in diameter than the IOL optics ${ }^{6}$. During the surgery, the IOL optic is brought through the CCC anteriorly to achieve a reverse rhexis fixation. This technique is useful when the IOL is placed in the capsular bag, but a posterior capsule tear occurs as soon as the IOL is placed in the bag or is noticed or extends after IOL placement. Therefore, the IOL fixation is not considered stable[7].

The main advantage of reverse optic capture is the possibility of using a single-piece IOL. Currently, these are the most common type of lenses applied in our operation room and are readily available. The injector system and incision size remain constant, while the reverse optic capture cannot be performed if the capsulotomy is too big to capture the optic effectively. Such an issue may occur in case of a manually performed CCC. In reverse optic capture, although the haptics are at a distance from the iris, the optic edge is anterior to the capsule and, therefore, close to the iris plane. This could predispose some patients to iris chafe, pigment dispersion, and Uveitis-Glaucoma-Hyphema (UGH) syndrome. It is important to monitor patients for these issues so that any problem can be addressed as soon as possible[7].

Another potential disadvantage highlighted by Dr. Jason J. Jones is the remaining fibrotic posterior capsular opacification caused by the optic not serving to separate the anterior and the residual posterior capsule. Posterior capsular YAG laser capsulotomy can and should be applied in cases of opacification occurrence[7].

In conclusion, isolated lens posterior capsule rupture after blunt eye injury is a rare complication and demands a special surgical management strategy in order achieve good visual outcome.

Research ethics and polices:

Conflict of interest:

Authors declare no potential conflicts of interest.

Acknowledgements:

No acknowledgments.

Prior publication:

This manuscript has not been published or presented elsewhere in part or in entirety and is not under consideration by another journal.

Permissions:

We hereby transfer, assign, or otherwise convey all copyright ownership, including any and all rights incidental thereto, exclusively to the journal, in the event that such work is published by the journal.

Declaration of patient consent

The authors certify that they have obtained all appropriate patient consent forms. In the form the patient has/have given his/her consent for his/her images and other clinical information to be reported in the journal. The patients understand that their names and initials will not be published and due efforts will be made to conceal their identity.

Author contribution:

Ēriks Elksnis: Corresponding author, literature review, data collection.

Juris Vanags: Manuscript revision. 
Eva Dručka: Literature review, data collection.

Oskars Gertners: Data collection.

Guna Laganovska: Final manuscript revision and approval.

References:

1 Lee SI, Song HC. A case of isolated posterior capsule rupture and traumatic cataract caused by blunt ocular trauma. Korean J Ophthalmol. 2001 Dec;15(2):140-4.

2 Wan W, Hu K, Ji Y, Li C. Management of Traumatic Cataract with Posterior Capsular Rupture: A Case Report and In Vitro Model Study. Case Rep Ophthalmol Med. 2017;2017:4230657.

3 Mansour AM, Jaroudi MO, Hamam RN, Maalouf FC. Isolated posterior capsular rupture following blunt head trauma. Clin Ophthalmol. 2014;8:2403-7.

4 Por YM, Chee S-P. Implantation of foldable intraocular lens with anterior optic capture in isolated posterior capsule rupture. J Cataract Refract Surg. 2006 May;32(5):707-8.

$5 \mathrm{Li}$ KK, Groenewald C, Wong D. Management of traumatic posterior capsular rupture: Corneal approach with high speed vitrector. J Cataract Refract Surg. 2005;31(8). Available from: https://journals.lww.com/jcrs/Fulltext/2005/08000/Management_of_traumatic_posterior_capsular.55.aspx

6 Simões PS, Ferreira TB. Iris-fixated intraocular lenses for ametropia and aphakia. Med hypothesis, Discov Innov Ophthalmol J. 2014;3(4):116-22.

7 Weiner G. Reverse Optic Capture After Posterior Capsular RuptureTitle. EyeNet Mag. 2016Available from: https://www.aao.org/eyenet/article/reverse-optic-capture-after-posterior-capsular-rup

Figure legends:

Figure 1. Slit lamp image of isolated posterior capsule rupture.

Figure 2. Slit lamp image of reverse optic capture.

Figure 3. Optical coherence tomography anterior segment scan imaging IOL optic before anterior capsule.

Figure 4. Optical coherence tomography anterior segment scan imaging IOL haptic on the right heading beneath anterior capsule. 

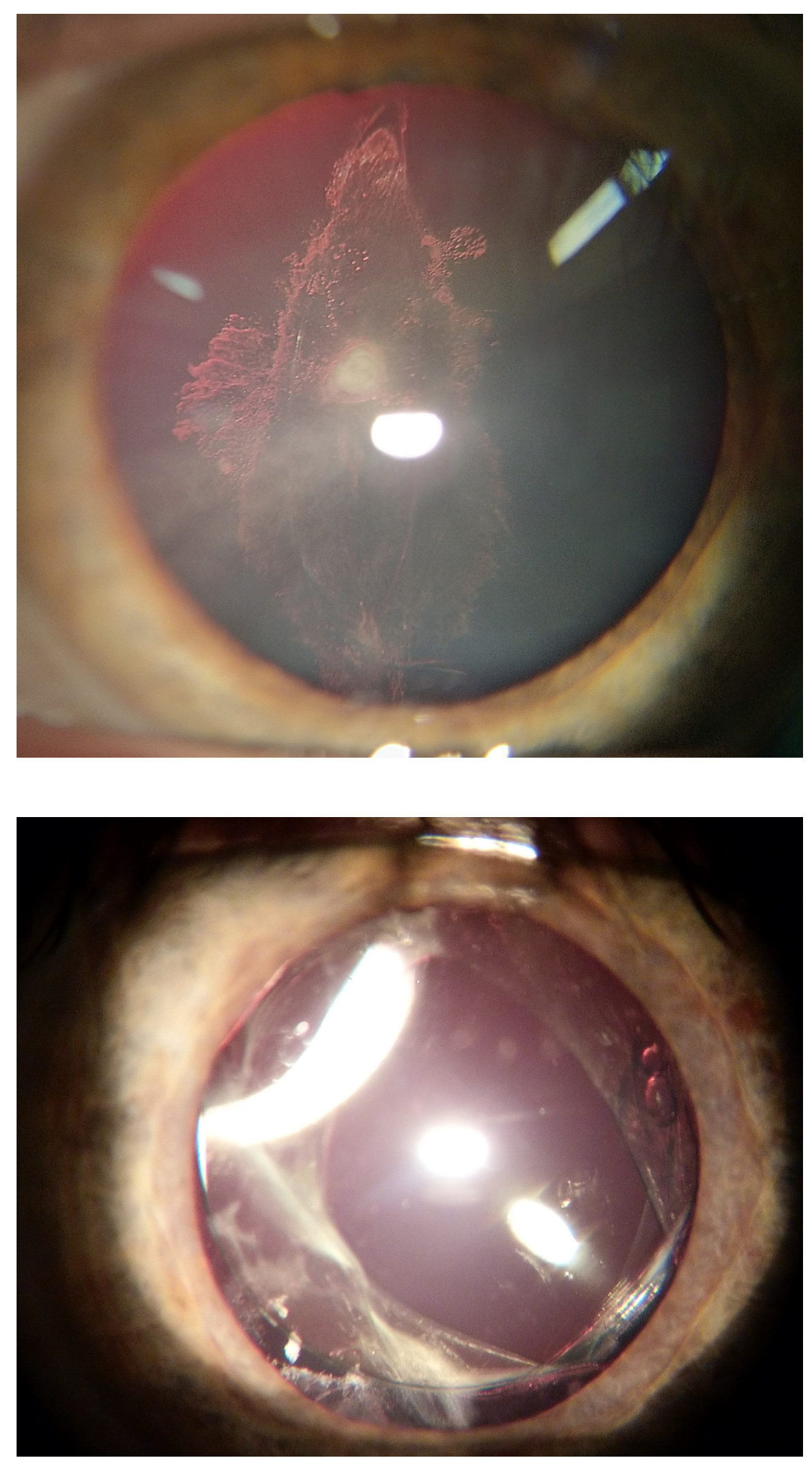

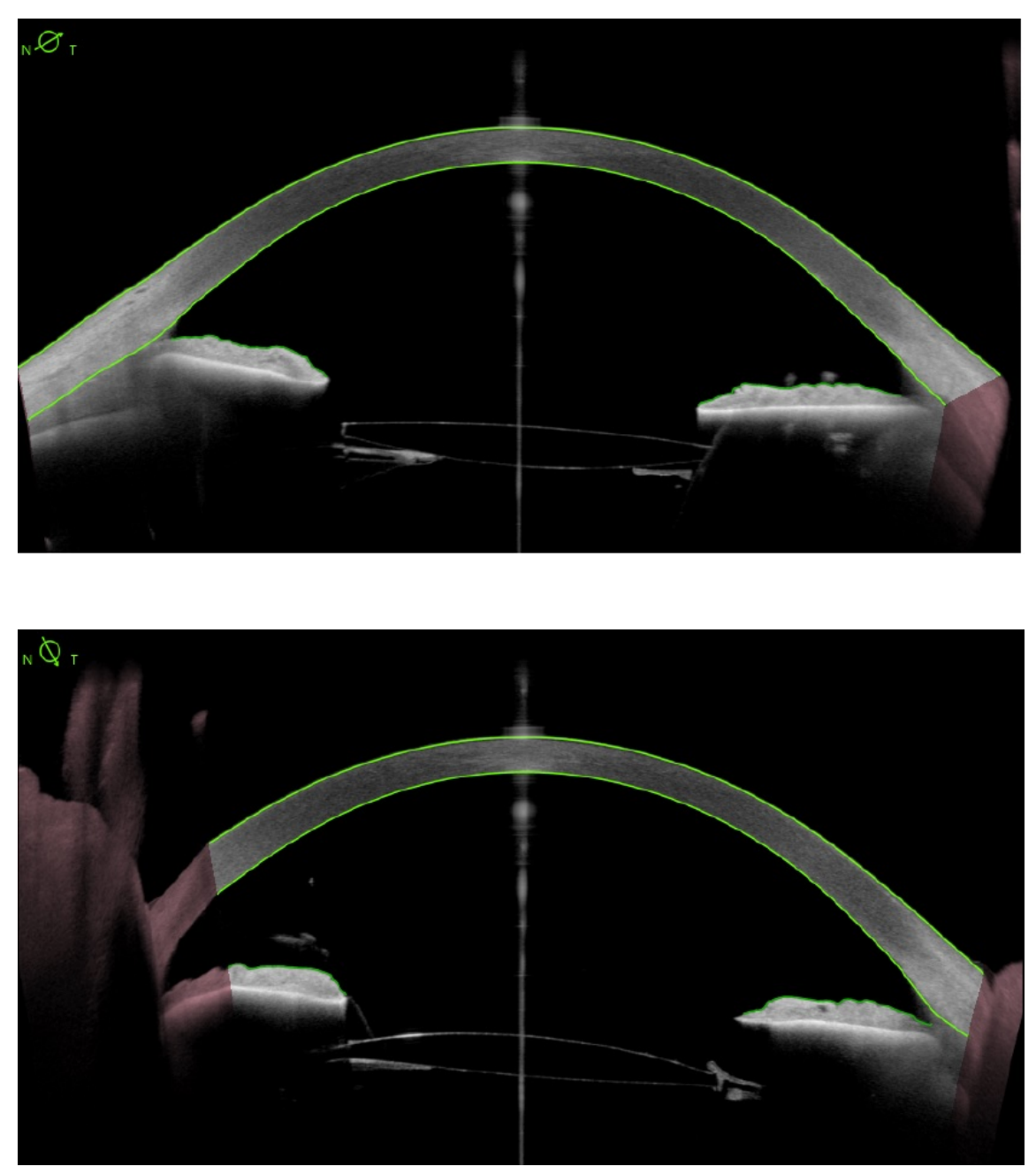